\title{
Measuring the bending of asymmetric planar EAP structures
}

\author{
Florian M. Weiss ${ }^{\mathrm{a}}$, Xue Zhao ${ }^{\mathrm{a}}$, Peter Thalmann ${ }^{\mathrm{a}}$, Hans Deyhle ${ }^{\mathrm{a}}$, Prabitha Urwyler ${ }^{\mathrm{a}}$, Gabor Kovacs ${ }^{\mathrm{b}}$, \\ and Bert Müller* \\ ${ }^{a}$ Biomaterials Science Center, University of Basel, c/o University Hospital, 4031 Basel, Switzerland; \\ ${ }^{\mathrm{b}}$ Swiss Laboratories for Materials Science and Technology, 8600 Dübendorf, Switzerland.
}

\begin{abstract}
The geometric characterization of low-voltage dielectric electro-active polymer (EAP) structures, comprised of nanometer thickness but areas of square centimeters, for applications such as artificial sphincters requires methods with nanometer precision. Direct optical detection is usually restricted to sub-micrometer resolution because of the wavelength of the light applied. Therefore, we propose to take advantage of the cantilever bending system with optical readout revealing a sub-micrometer resolution at the deflection of the free end. It is demonstrated that this approach allows us to detect bending of rather conventional planar asymmetric, dielectric EAP-structures applying voltages well below $10 \mathrm{~V}$. For this purpose, we built $100 \mu \mathrm{m}$-thin silicone films between $50 \mathrm{~nm}$-thin silver layers on a $25 \mu \mathrm{m}$-thin polyetheretherketone (PEEK) substrate. The increase of the applied voltage in steps of $50 \mathrm{~V}$ until $1 \mathrm{kV}$ resulted in a cantilever bending that exhibits only in restricted ranges the expected square dependence. The mean laser beam displacement on the detector corresponded to $6 \mathrm{~nm}$ per volt. The apparatus will therefore become a powerful mean to analyze and thereby improve low-voltage dielectric EAP-structures to realize nanometer-thin layers for stack actuators to be incorporated into artificial sphincter systems for treating severe urinary and fecal incontinence.
\end{abstract}

Keywords: Micro-cantilever, polyetheretherketone (PEEK), low-voltage dielectric electro-active polymer, artificial sphincter, silicone, silver contact, reflective gold layer thickness, Stoney formula

\section{INTRODUCTION}

More than a century ago G. G. Stoney described the bending of a metallic beam due to surface stress, which a thin layer of another metal created [1]. Nickel deposited by electrolysis induced the bending of steel rules. Stoney was able to describe the observation quantitatively. This description of the cantilever bending became the basis of a series of micro and nano electromechanical systems (MEMS and NEMS, respectively) for sensing applications and of the single crystal bending upon (sub-monolayer) heteroepitaxy resulting from the lattice mismatch.

One of the most prominent examples is the invention of scanning probe microscopy including scanning tunneling microscopy (STM) and atomic force microscopy (AFM), cp. for example [2, 3]. The experimental setup of G. Meyer and N. M. Amer is based on the detection of the laser beam from the free end of the cantilever opposite to the detecting tip. The laser beam generates a voltage on the two-dimensional position-sensitive detector (PSD), which depends on the position of the reflected laser beam.

The micro-cantilever bending approach is usually termed 'static mode'. Based on this principle many sensors were realized. J. K. Gimzewski et al. built a micro-calorimeter with a sensitivity of about one picoJoule [4]. Using a wellestablished single crystal silicon cantilever coated on one side by aluminum and platinum, the catalytic reaction of oxygen and hydrogen molecules was detected via the resulting heat flow. As the platinum catalyzed reaction takes place, heat is transferred through the two layers provoking the bimetallic effect. This effect causes the cantilever to bend and the related shift of the reflected laser beam on the PSD is easily determined.

The costly silicon-technology-based cantilevers can be replaced by low-cost polymer cantilevers, which, for the same thickness, show similar or even higher sensitivity because of the lower Young's modulus. Recently, for example, Urwyler et al. have prepared polypropylene micro-cantilever arrays taking advantage of injection molding [5, 6] and successfully applied them to detect bio-medically relevant metal concentrations and to identify DNA-fragments on the functionalized micro-cantilevers [7].

*bert.mueller@unibas.ch; phone +4161265 9660; fax +4161265 9699; www.bmc.unibas.ch

Electroactive Polymer Actuators and Devices (EAPAD) 2013, edited by Yoseph Bar-Cohen,

Proc. of SPIE Vol. 8687, 86871X - @ 2013 SPIE · CCC code: 0277-786X/13/\$18 · doi: 10.1117/12.2009355

Proc. of SPIE Vol. 868786871 X-1 
The approach can also be applied to 'thick films'. Köser et al. [8], for example, seeded a silicon cantilever on one side with a confluent monolayer of biological cells. The adherent fibroblasts deform the underlying substrate because of their contractile forces necessary for cell migration. By biochemically detaching the cells from the silicon micro-cantilever the relaxation of the cantilever was detected and the mean contractile force per cell derived. Also here, the Stoney formula was used. The applicability of the Stoney formula remains, however, unclear.

In this study, we claim that the cantilever bending method is a powerful and sensitive tool to directly measure the force and the related stress exerted by an EAP-microstructure on top of a polymer cantilever increasing the applied voltage in steps of $50 \mathrm{~V}$. Such a device should become a standard instrument for the improvement of the fabrication process to realize dielectric actuators for the low-voltage regime as required for artificial sphincters $[9,10]$ to successfully treat severe urinary and fecal incontinence.

\section{EXPERIMENTAL}

\subsection{Electrically active polymer structures}

Polyetheretherketone (PEEK) films (APTIV 2000, Victrex, Lancashire, UK) $25 \mu \mathrm{m}$ thin were cut to $4 \mathrm{~cm} \times 4 \mathrm{~cm}$ squares. The film pieces were rinsed in acetone to clean their surfaces. Subsequently, a $50 \mathrm{~nm}$-thin silver layer (Lesker, Columbus, Georgia, USA) was deposited on the smooth side under ultrahigh vacuum conditions. The sputtering conditions in the ATC 1500-F system (AJA International Inc., North Scituate, MA, USA) corresponded to 0.1 mbar argon atmosphere (Messer, Lenzburg, Switzerland) with a flow of $49.5 \mathrm{sccm}$. During the sputtering time of 15 minutes a power of $200 \mathrm{~W}$ was applied.

Scotch tape was used to cover a part of the electrode to build the electrical contact in the final stage (cp. Figure 1). Then, polydimethylsiloxane (PDMS) (Elastosil 745 A/B, Wacker Chemie AG, Munich, Germany) was mixed in a 1:1 ratio (component $\mathrm{A}$ and $\mathrm{B}$ ) and distributed on the silver layer using doctor blading. To crosslink the PDMS films, the structures were put into an oven and heated at a temperature of $80^{\circ} \mathrm{C}$ for a period of 90 minutes. The resulting PDMSlayer had a thickness of $(102 \pm 10) \mu \mathrm{m}$ measured on cross sections by means of conventional secondary electron microscopy. After PDMS-crosslinking the sheets were sputtered with another $50 \mathrm{~nm}$-thin silver film for the second electrode. Finally, the masking tape was carefully removed.

\subsection{Cantilever preparation}

The cantilevers were cut to obtain a free end with a length of $15 \mathrm{~mm}$ and a width of $3 \mathrm{~mm}$. The cantilevers were connected to the aluminum support plate on one end using double-side Scotch tape. The contacts were wired applying silver paint (Acheson Silver, Plano GmbH, Wetzlar, Germany).

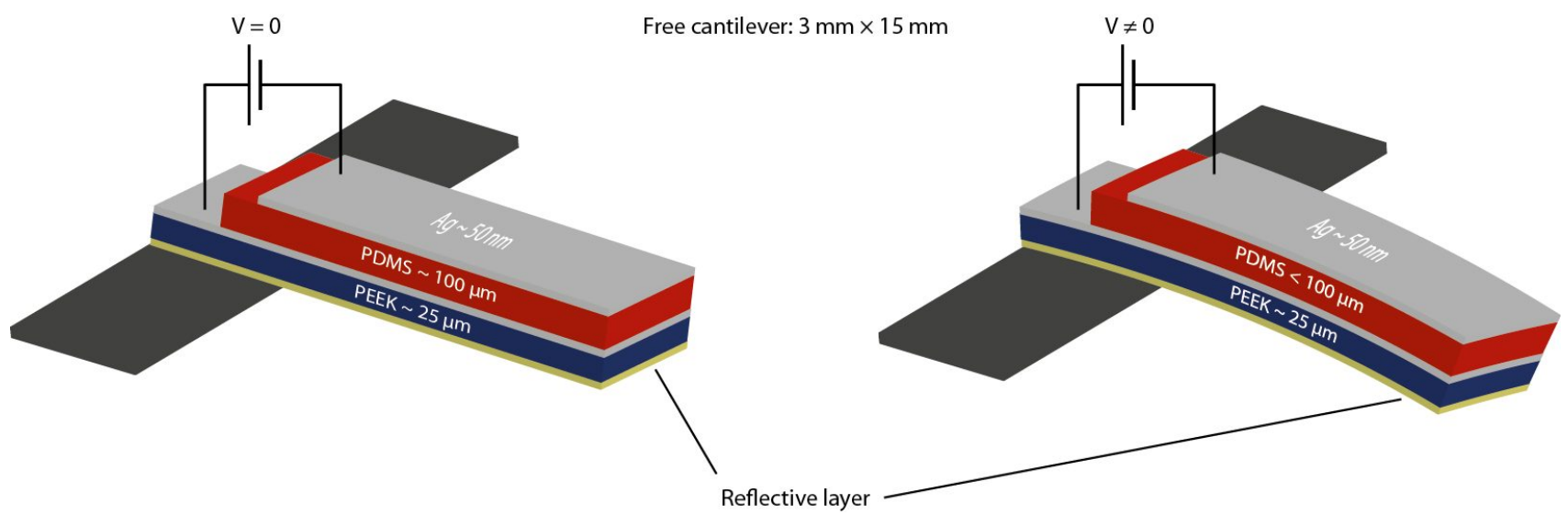

Figure 1. Without the application of a voltage the cantilever is nominally flat (left scheme). Because of the applied voltage the PDMS film is laterally stretched and deforms the anisotropic microstructure (right scheme). Therefore, the cantilever bending can be measured as the function of the applied voltage to quantify the performance of the EAP structure. 


\subsection{Cantilever bending calibration and measurement}

The system to measure the cantilever bending is built on a rotatable frame that holds the laser (He-Ne, $10 \mathrm{~mW}$, Uniphase, Mantca, CA, USA), the cantilever on its holder and the position-sensitive detection unit (PSD, 2L4SP, SiTek, Laser Components, Olching, Germany). Figure 2 displays the arrangement of the components including the laser beam path. The entire system is placed on an air-damped table (Stada, Vilnius, Lithuania) to reduce the negative impact of vibrations from the floor. The deflection of the vertically oriented cantilever was measured during the step-wise increase of the applied voltage provided from a Stanford Research System high-voltage power supply (PS310, GMP SA, Lausanne, Switzerland). Vertical arrangement was chosen to avoid cantilever bending by the gravity.

In order to achieve reasonable reflectivity of the laser beam on the free end of the cantilever, a piece of single crystalline silicon was used. As an alternative one can deposit a reflective gold layer with an optimized thickness of $28 \mathrm{~nm}$. This choice guarantees reasonable reflectivity for the wavelength of $633 \mathrm{~nm}$ of the HeNe laser [11].

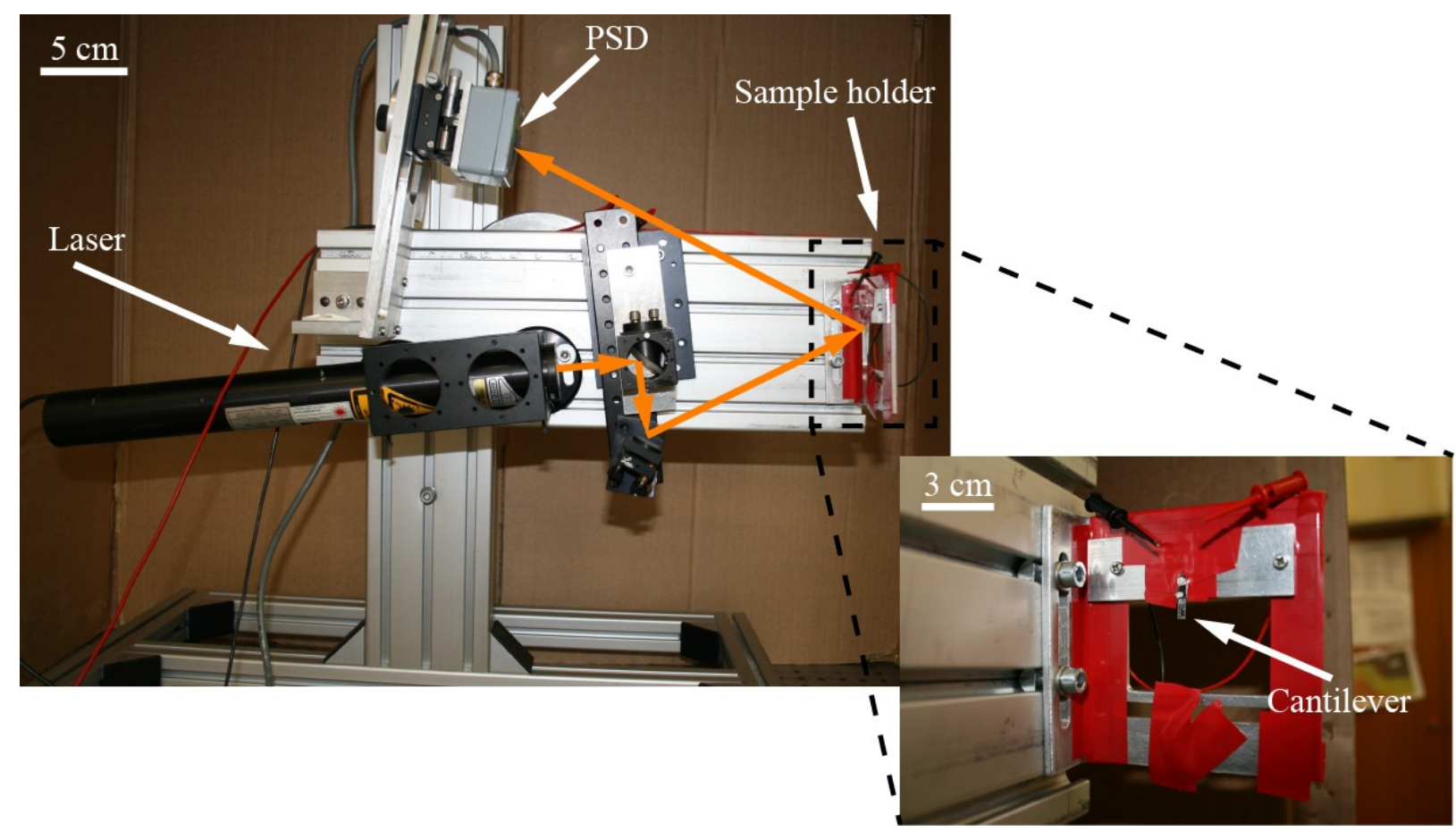

Figure 2. The laser beam (orange line) is directed to the free end of the cantilever and reflected towards the position sensitive detector (PSD). The aluminum frame can be rotated to calibrate the cantilever through gravity-induced bending.

\section{RESULTS}

Increasing the applied voltage from 0 to $1{ }^{\prime} 000 \mathrm{~V}$, the laser spot on the PSD moves by several micrometers, as shown by the data in Figure 3. The displacement is large in comparison with the spatial resolution of $41 \mathrm{~nm}$ of the entire system detected on the PSD unit. This value was derived from the fluctuations of the laser beam on the PSD without applying a voltage, i. e. from the standard deviation of 200 subsequently acquired data points. The data shown in Figure 3 indicate that the cantilever bending increases with about $6 \mathrm{~nm}$ per volt. Together with the spatial resolution of $41 \mathrm{~nm}$ this means that voltage steps below $7 \mathrm{~V}$ are not meaningful, and the choice to increase the applied voltage in steps of $50 \mathrm{~V}$ has been suitable.

The EAP structure has to be conditioned running five cycles, before one can observe a reproducible, almost identical behavior increasing or decreasing the voltage (cp. the two graphs of Figure 3). After the conditioning, the cantilever bending should follow the square dependence of the applied voltage (see below). Therefore, the data shown in the right part of Figure 3 are plotted in the adapted way in Figure 4. 
The Maxwell pressure $p$, i.e. the force $F$ per area $A$, can be written as:

$$
p=\varepsilon_{r} \varepsilon_{0}\left(\frac{U}{z}\right)^{2}
$$

with the applied voltage $U$, the layer thickness of the elastomer $z$ and the relative dielectric constants of the elastomer and of the vacuum $\varepsilon_{r}$ and $\varepsilon_{0}$, respectively. This pressure induces a surface stress on the PEEK cantilever. If the bonding between the EAP-structure and the PEEK-film is strong enough, the whole structure (EAP plus PEEK substrate) will be bent towards the EAP far side.

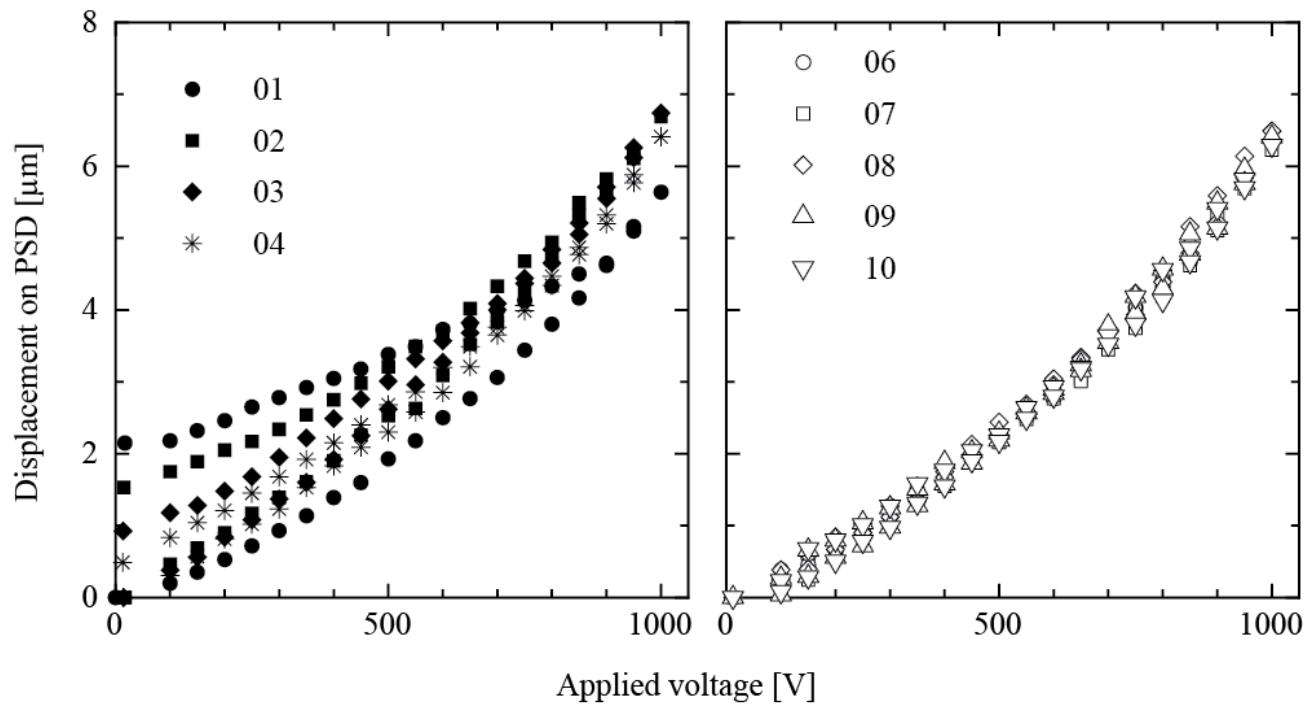

Figure 3. During the first five cycles the cantilever does not reach the starting position. From the sixth cycle onward the cantilever shows a reproducible behavior, when a voltage is applied.

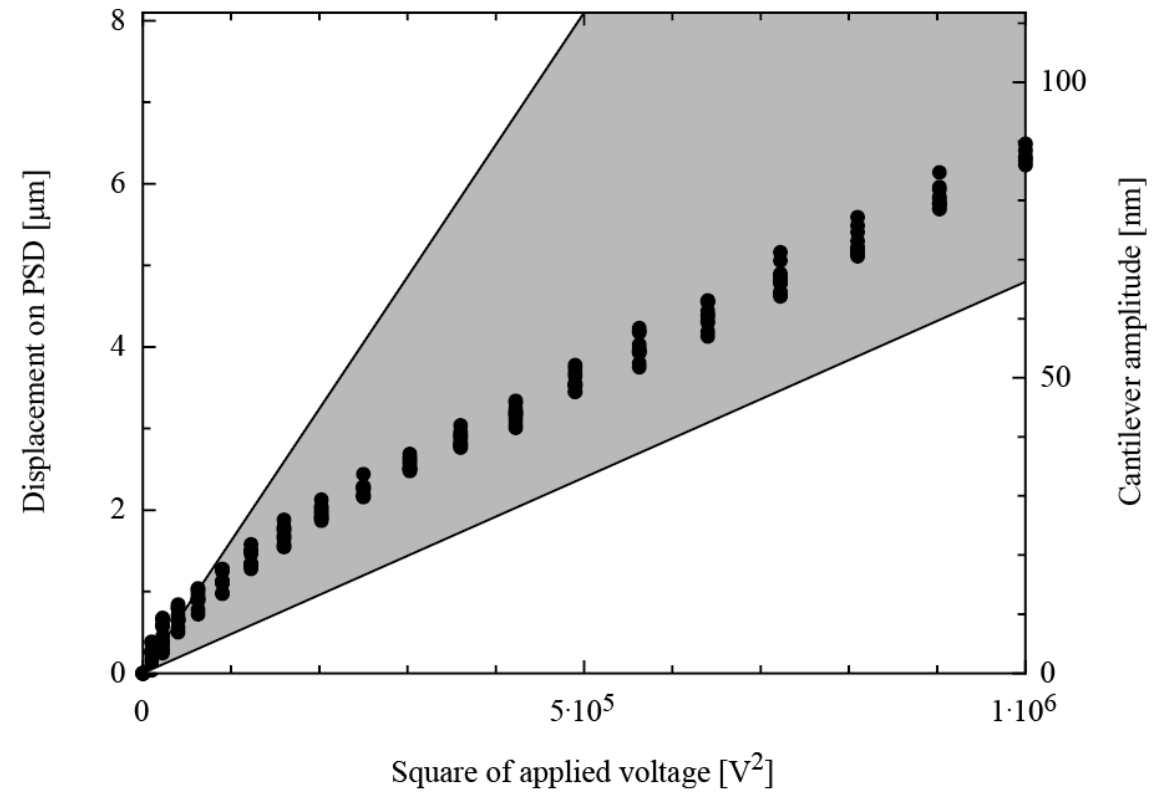

Figure 4. The bending of the cantilever with the EAP-structure on top does follow the square voltage dependence only in restricted ranges. These rather preliminary data, however, do not allow any conclusive statement on the nature of the measured data. 
It is rather unclear how the applied force $F$ affects the surface stress. One might reasonably expect that the induced inplane stress at the PDMS-PEEK junction is proportional to the applied pressure $p$. Furthermore, the elastic modulus of the PDMS $\left(E_{\mathrm{PDMS}}=750 \mathrm{kPa}\right)$ is more than three orders of magnitude smaller than the one of the PEEK substrate $\left(E_{\mathrm{PEEK}}=1.8 \mathrm{GPa}\right)$ so that the mechanics of the hybrid cantilever are dominated by the underlying PEEK.

Applying the Stoney formula to calculate the surface stress $\sigma$

$$
\sigma=\frac{E_{\text {PEEK }}}{6\left(1-v_{\text {PEEK }}\right)} * \frac{z_{\text {PEEK }}^{2}}{z_{P D M S} R}
$$

with $v$ the Poisson ratio of the PEEK substrate one finds that under our assumption that the curvature of the cantilever $\kappa=1 / R$ and, therefore, the displacement of the laser beam on the PSD is directly proportional to the square of the applied voltage, which agrees with data measured with an applied voltage higher than $400 \mathrm{~V}$ (cp. Figure 4).

Figure 4 includes the cantilever amplitude (see right ordinate), i. e. the change of the free end in position, which is directly proportional to the movement of the laser beam on the PSD. The presently used laser pointer method translates the spatial resolution on the PSD $(47 \mathrm{~nm})$ to a cantilever amplitude of $0.7 \mathrm{~nm}$.

\section{DISCUSSION}

Cantilever bending belongs to the established techniques that show extremely high sensitivity and are, thus, well suited to detect deformations as the result of atomic or molecular interactions [2-4, 7, 8, 12-19]. In comparison with the established examples of micro-cantilever bending the present approach shows some key differences. First, due to the electrostatic force the cantilever thickness decreases by increasing the applied voltage. In the present case, the thickness decrease per $\mathrm{kV}$ corresponds to about $700 \mathrm{~nm}$. Second, the mechanical properties of the EAP-structure might also be a function of the applied voltage. Third, the question arises whether the approach of Stoney can be meaningfully applied to EAP-structures, which are more than a factor of four thicker than the underlying PEEK substrate. Note the film thickness is in many cases as described in the references listed above negligible with respect to the cantilever thickness.

To this end, we have to be aware that the translation of the Maxwell pressure into the surface stress on the PEEKcantilever and, therefore, the cantilever bending of the asymmetric EAP-structure is not yet understood. Further theoretical and experimental work will be required to quantitatively describe the rather preliminary data presented in Figures 3 and 4.

\section{CONCLUSION}

The cantilever beam bending approach is a powerful and comparably cost-efficient mean to detect the deformation of EAP micro- and nanostructures, which results in the bending of the anisotropic system EAP-structure - micro-cantilever. Polymer films such as PEEK are well suited for the micro-cantilevers.

\section{ACKNOWLEDGMENT}

The financial support of the Swiss National Science Foundation (project 200021-135496) is gratefully acknowledged.

\section{REFERENCES}

[1] G. G. Stoney, "The tension of metallic films deposited by electrolysis," Proceedings of the Royal Society of London. Series A, 82(553), 172-175 (1909).

[2] G. Binnig, C. F. Quate, and C. Gerber, “Atomic force microscope,” Physical Review Letters, 56(9), 930-933 (1986).

[3] G. Meyer, and N. M. Amer, "Novel optical approach to atomic force microscopy," Applied Physics Letters, 53(12), 1045-1047 (1988). 
[4] J. K. Gimzewski, C. Gerber, E. Meyer et al., "Observation of a chemical reaction using a micromechanical sensor," Chemical Physics Letters, 217(5, 6), 589-594 (1994).

[5] P. Urwyler, A. Pascual, P. M. Kristiansen et al., "Mechanical and chemical stability of injection-molded microcantilevers used for sensing," Journal of Applied Polymer Science, 127(4), 2363-2370 (2013).

[6] P. Urwyler, H. Schift, J. Gobrecht et al., "Surface patterned polymer micro-cantilever arrays for sensing," Sensors and Actuators A, 172, 2-8 (2011).

[7] P. Urwyler, J. Köser, H. Schift et al., "Nano-mechanical transduction of polymer micro-cantilevers to detect biomolecular interactions," Biointerphases, 7, 6 (2012).

[8] J. Köser, S. Gaiser, and B. Müller, "Contractile cell forces exerted on rigid substrates," European Cells and Materials, 21, 479 - 487 (2011).

[9] B. Müller, H. Deyhle, S. Mushkolaj et al., "The challenges in artificial muscle research to treat incontinence," Swiss Medical Weekly, 139(41-42), 591-595 (2009).

[10] F. Weiss, H. Deyhle, G. Kovacs et al., "Designing micro- and nanostructures for artificial urinary sphincters," Proceedings of SPIE, 8340, 83400A1 (2012).

[11] X. Zhao, P. Urwyler, and B. Müller, "Optimization of the optical readout of PEEK cantilevers," European Cells and Materials, 23(2), 40 (2012).

[12] Y. Arntz, J. D. Seelig, H. P. Lang et al., "Label-free protein assay based on a nanomechanical cantilever array," Nanotechnology, 14, 86-90 (2003).

[13] G. Y. Chen, T. Thundat, E. A. Wachter et al., "Adsorption-induced surface stress and its effects on resonance frequency of microcantilevers," Journal of Applied Physics, 77(8), 3618-3622 (1995).

[14] J. Fritz, M. K. Baller, H. P. Lang et al., "Translating biomolecular recognition into nanomechanics," Science, 288(5464), 316-318 (2000).

[15] J. Fritz, M. K. Baller, H. P. Lang et al., "Stress at the solid-liquid interface of self-assembled monolayers on gold investigated with a nanomechanical sensor," Langmuir, 16(25), 9694-9696 (2000).

[16] B. Ilic, D. Czaplewski, H. G. Craighead et al., "Mechanical resonant immunospecific biological detector," Applied Physics Letters, 77(3), 450-452 (2000).

[17] H. P. Lang, M. K. Baller, R. Berger et al., "An artificial nose based on a micromechanical cantilever array," Analytica Chimica Acta, 393(1-3), 59-65 (1999).

[18] A. M. Moulin, S. J. O'Shea, R. A. Badley et al., "Measuring surface-induced conformational changes in proteins," Langmuir, 15(26), 8776-8779 (1999).

[19] V. Tabard-Cossa, M. Godin, L. Y. Beaulieu et al., "A differential microcantilever-based system for measuring surface stress changes induced by electrochemical reactions," Sensors and Actuators B: Chemical, 107(1), 233-241 (2005). 